\title{
Relación entre bronquiolitis aguda con factores climáticos y contaminación ambiental
}

\author{
Alejandra Zamorano $\mathbf{W}^{1}$, Sonia Márquez $\mathrm{U}^{1}$, \\ Juan Luis Aránguiz $\mathbf{R}^{1}$, Paula Bedregal $\mathbf{G}^{2}$, \\ Ignacio Sánchez D1.
}

\section{Association of acute bronchiolitis with environmental variables}

Background: Annually, acute bronchiolitis (AB) occurrence peaks during winter and is probably associated with air pollution. Aim: To relate the number of ambulatory consultations, emergency and hospital admission due to AB with climatic factors and air pollution. Patients and methods: Patients of less than 1 year old with AB that consulted to outpatient clinics, the emergency room or were admitted to the Pediatrics ward of the Catholic University Hospital, were enrolled. Information about respiratory syncytial virus (RSV) was obtained from the Catholic University Medical Investigation Center. Indices of air pollution such as particulate matters of less than 10 microns $/ \mathrm{m}^{3}$ (PM 10), of less than 2.5 microns $/ \mathrm{m} 3$ ( $\mathrm{PM}$ 2.5), $\mathrm{CO}, \mathrm{SO}_{3}$ and $\mathrm{O}_{3}$ were obtained from the Metropolitan Environmental Service. Temperature, humidity and precipitations were obtained from the Chilean Meteorological Service. Results: Ninety nine consultations in out patient clinics and 442 in emergency rooms were collected (55\% male, mean age 4.8 months). One hundred fifty two were admitted (34.4\%). Thirty percent of children consulting in emergency rooms were younger than 3 months and $43 \%$ of them were hospitalized. The RSV study was made in 307 patients and $52 \%$ were positive. There was a higher rate of hospital admissions among RSV positive than RSV negative patients (52.5 and $22 \%$ respectively, $\mathrm{p}<0.001$ ). No association between environmental variables or air pollution and the number of consultations was observed. Young age and smoking inside the household were the main risk factors for hospital admission due to acute bronchiolitis. Conclusions: Environmental variables did not influence the number of cases of acute brochiolitis. Young age and exposure to tobacco smoke were risk factors for hospital admission (Rev Méd Chile 2003; 131: 1117-22).

(Key Words: Air pollutants, environmental; Bronchiolitis; Tobacco smoke pollution)

Recibido el 3 de enero, 2003. Aceptado en versión corregida el 6 de agosto, 2003.

Sección Respiratorio Pediátrico, Departamentos de Pediatría ${ }^{1}$ y Salud Pública ${ }^{2}$, Facultad de Medicina, Pontificia Universidad Católica de Chile.

Correspondencia a: Dr. Ignacio Sánchez. Lira 85, $5^{\circ}$ piso. Departamento Pediatría, Facultad de Medicina. Fono: 3543887. Fax: 6384307. E mail: igsan@med.puc.cl 
as infecciones respiratorias en los menores de ـ1 año, específicamente la bronquiolitis aguda (BA), ya sea por virus sincicial respiratorio (VRS) 0 por otra etiología, son un problema de salud importante tanto a nivel nacional como mundial. Esta es la causa más frecuente de morbilidad, consulta, tanto a nivel primario como de servicios de urgencia y hospitalización en períodos de otoño e invierno. Esta fluctuación estacional sugiere que factores ambientales como el frío, la humedad y precipitaciones pueden jugar un rol en la incidencia de enfermedades respiratorias, asociado a la presencia de brotes estacionales de virus respiratorios que año tras año participan de las epidemias de cuadros respiratorios ${ }^{1-3}$.

La contaminación ambiental, tanto de elementos particulados como emisiones de gases, es problema en muchas grandes ciudades del mundo, dentro de las cuales Santiago de Chile ocupa un lugar destacado. Estudios previos han demostrado una correlación significativa entre el aumento de la concentración de partículas en el aire con disminución de la función pulmonar de niños ${ }^{4}$, encontrando que la contaminación ambiental es capaz de gatillar crisis bronquiales obstructivas en niños con síntomas respiratorios ${ }^{5}$. El aumento en el número de consultas y hospitalizaciones también se ha visto relacionado con el aumento de los niveles de elementos particula$\operatorname{dos}^{6}$. Por otro lado, hay trabajos que no encuentran que el factor contaminación juegue un rol importante en la producción de crisis asmáticas ${ }^{7}$. Estudios recientes confirman la asociación entre mortalidad aguda y aumento del PM10 y $\mathrm{SO}_{2}$, principalmente en personas mayores y con patología respiratoria preexistente, siendo importante clarificar cuáles son los componentes del PM10 más importantes en esta asociación con mortali$\operatorname{dad}^{8,9}$.

La contaminación intradomiciliaria es otro factor que se ha relacionado con las enfermedades respiratorias en niños pequeños, principalmente los elementos de calefacción y tabaquismo domiciliario, especialmente materno ${ }^{10-13}$. Son pocos los estudios, tanto a nivel nacional como extranjero, que analizan la relación entre todos estos factores: climáticos, contaminación intra y extradomiciliaria, presencia de virus con el aumento en consulta y hospitalización por enfermedades respiratorias 2,3 .
El objetivo del presente estudio fue estudiar la asociación entre factores climáticos (temperatura, humedad relativa, precipitaciones) y de contaminación intra y extradomiciliaria (métodos de calefacción, tabaquismo pasivo y elementos particulados, $\mathrm{SO}_{2}, \mathrm{O}_{3}$, y CO respectivamente) con la frecuencia de presentación de bronquiolitis aguda.

\section{MATERIAL Y MÉTODO}

Pacientes. Se ingresaron, desde el 21 de mayo al 30 de septiembre del año 2001, los pacientes con BA menores de 1 año que consultaron en un Centro Ambulatorio, Servicio de Urgencia (SU) o que fueron hospitalizados en el Servicio de Pediatría del Hospital Clínico de la Universidad Católica, en la ciudad de Santiago de Chile. Se definió BA como el primer episodio bronquial obstructivo en menores de 12 meses, independiente de la etiología de éste. Se realizó una encuesta telefónica consultando además factores como contaminación intradomiciliaria, (calefacción, tabaquismo) y nivel educacional de los padres.

Estudio virológico. En el mismo período se obtuvo información de los virus respiratorios circulantes en la Región Metropolitana por el Centro de Investigaciones Médicas (PUC), correspondiente a la vigilancia epidemiológica de virus respiratorios. Se realizó además en algunos pacientes un estudio etiológico viral, poniéndose mayor énfasis en la presencia de virus respiratorio sincicial (VRS). La muestra se obtuvo mediante hisopado nasofaríngeo, realizándose inmunofluorescencia o test pack.

Contaminación ambiental. Se consideró como índice de contaminación las partículas en suspensión $\leq 10$ micrones $/ \mathrm{m}^{3}$ (PM10) y las $\leq 2,5$ micrones/ $\mathrm{m}^{3}$ (PM2.5). Los valores de elementos contaminantes (PM10, $\mathrm{PM} 2.5, \mathrm{CO}, \mathrm{SO}_{3}, \mathrm{O}_{3}$ ) se obtuvieron a través del Servicio de Salud del Medio Ambiente (SESMA) en forma diaria, consignando para cada paciente el valor según la comuna de residencia. Se contó con la información de 8 centros de medición para contaminación distribuidos en las comunas de Providencia, Independencia, Cerrillos, El Bosque, La Florida, Las Condes, Santiago y Pudahuel (para PM2.5 sólo se registran en los últimos cuatro centros de medición). 
Factores climáticos. La información de factores climáticos (temperatura, humedad, precipitaciones) se obtuvo de la Dirección Meteorológica de Chile, con 4 centros de medición: Quinta Normal, Pudahuel, Cerrillos, Tobalaba, además de la Estación de Avenida La Paz, aportada por el SESMA. Esta información se obtuvo en forma diaria.

Estadística. Se realizó un análisis descriptivo de las variables en estudio y de correlación entre el número de casos, presencia de virus sincicial respiratorio (VRS) y las variables ambientales. Además se realizó un análisis de serie de tiempo, análisis de regresión logística (Backward y Enter $^{11}$ en búsqueda de variables explicatorias de hospitalización.

\section{RESUlTADOS}

Entre el 21 de mayo al 30 de septiembre de 2001 hubo un total de 541 pacientes menores de 1 año atendidos por cursar un episodio de BA; 99 fueron atendidos en un centro de pediatría ambulatoria y 442 en el SU, de las cuales se hospitalizaron 152 (34,4\%). La distribución por sexo fue de $45 \%$ mujeres y $55 \%$ hombres. La edad promedio fue 4,8 meses (2 días - 1 año, DS 3,15 meses). Del total de las consultas en el SU, 30,3\% fueron de menores de 3 meses, de los cuales se hospitalizó el 43\%. Se estudió VRS en 307 pacientes, de los cuales fue positivo ( + ) en 52,1\%; en 11 no se obtuvo el resultado (3,6\%). Los pacientes VRS (+) se hospitalizaron $52,5 \%$ vs $22 \%$ de los VRS (-) independiente de la edad ( $p=0,0003)$

En la Figura 1 se observa el comportamiento a través de las semanas estudiadas de PM10, PM2.5, diferencia de temperatura y número de casos. Al observar las fluctuaciones de las variables, destaca cierta concordancia entre el aumento de número de casos y aumento de PM10 y PM2.5, la que no resultó ser significativa. En la Tabla 1 se observan las correlaciones simples entre las variables ambientales, número de casos y VRS (+). Las correlaciones simples entre las variables ambientales y número de casos no demostraron asociación significativa, como tampoco con VRS (+). Sí se encontró una asociación significativa entre aumento de PM10, PM2.5 y menor temperatura, como también entre PM10 y PM2.5.

El análisis de autocorrelación de primer orden y parcial, que nos permite conocer el comportamiento en el tiempo de una variable sobre sí misma, de PM10, PM2.5 y diferencia de temperatura no mostró dependencia, es decir el valor presente en un día no influye en los valores posteriores. Por el contrario, sí se observó estacionalidad con el número de casos, observándose un

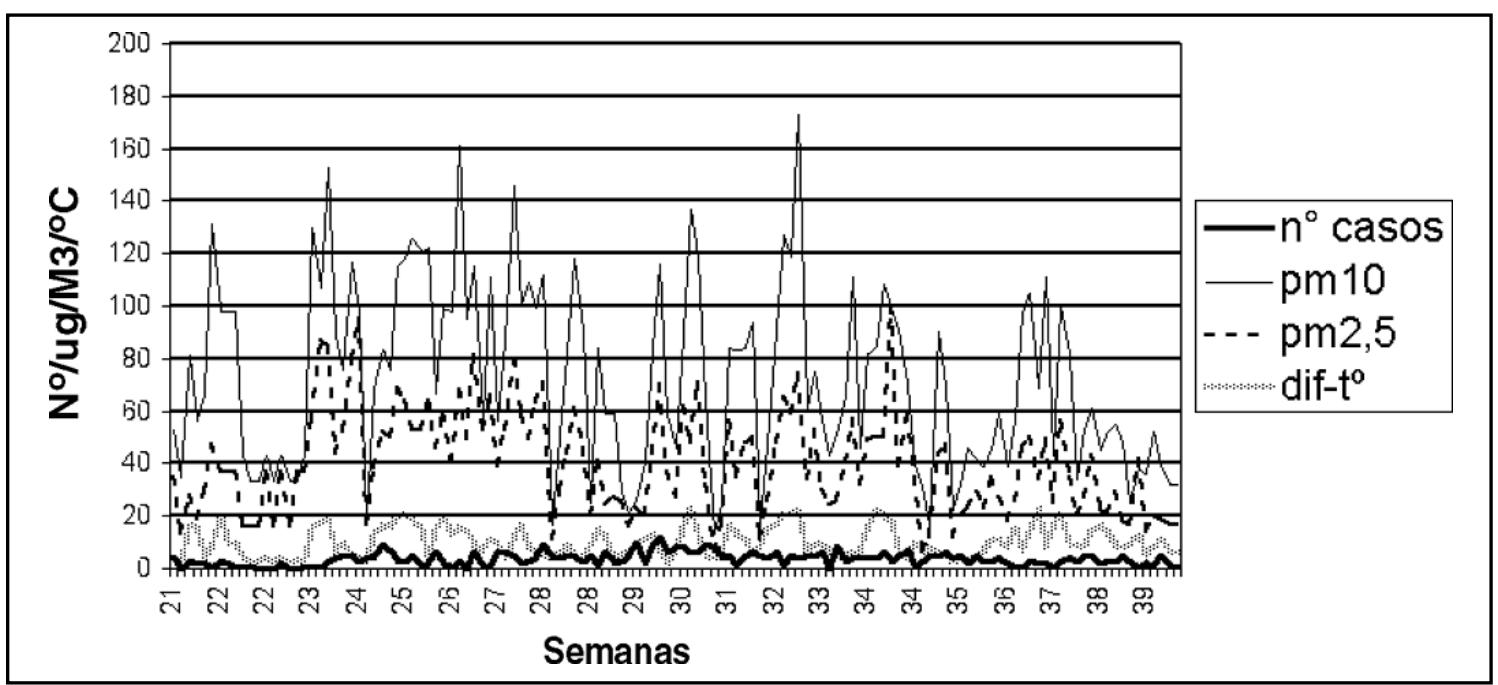

FiguRA 1. Serie de tiempo: se observa el comportamiento a través de las semanas estudiadas de PM10, PM2.5, diferencia de temperatura y número de casos. Destaca cierta concordancia entre el aumento de número de casos y aumento de PM10 y PM2.5, la que no resultó ser significativa. 
Tabla 1. Correlaciones simples entre variables ambientales y número de casos

\begin{tabular}{|lccc|}
\hline & $\begin{array}{c}\text { Coeficiente } \\
\text { Correlación } \mathrm{r}\end{array}$ & Valor $\mathrm{p}$ & $\begin{array}{c}\text { Intervalo confianza 95\% } \\
\text { para } \mathrm{r}\end{array}$ \\
\hline PM10 y № de casos & $-0,0074$ & 0,9331 & $(-0,1780 ; 0,1637)$ \\
PM2.5 y № de casos & 0,0299 & 0,7336 & $(-0,1417 ; 0,1998)$ \\
Diferencia To y № de casos & $-0,0206$ & 0,8149 & $(-0,1908 ; 0,1508)$ \\
PM10 y PM2.5 & 0,8806 & 0,0001 & $(0,8354 ; 0,9139)$ \\
PM10 y diferencia de Tㅜ & 0,4255 & 0,0001 & $(0,2746 ; 0,5559)$ \\
PM2.5 y diferencia de To & 0,3465 & 0,0001 & $(0,1866 ; 0,4884)$ \\
\hline
\end{tabular}

PM10: material particulado $\leq 10 \mathrm{um} / \mathrm{m}^{3}$

№ de casos: número de casos

PM2.5: material particulado $\leq 2.5 \mathrm{um} / \mathrm{m}^{3}$

Diferencia de $\mathrm{T}^{\circ}$ : diferencia entre temperatura máxima y mínima

patrón cíclico en su comportamiento, con variaciones diarias. No se logró desarrollar un modelo predictivo (Autoregressive integrated moving average: ARIMA) del número de casos de BA.

Como predictor de riesgo de hospitalización se ingresaron en un modelo de regresión logística la edad del niño, el antecedente de tabaquismo materno o de otro miembro de la familia, la educación materna y paterna y los factores ambientales. Las dos variables que ingresan finalmente en el modelo son ser menor de 3 meses $(\mathrm{OR}=2,19 ; \mathrm{p}=0,0001)$, como factor de riesgo y el no consumo de tabaco intradomiciliario por los miembros del hogar ( $\mathrm{OR}=0,5447 ; \mathrm{p}=0,0071)$ como protector de hospitalización.

\section{Discusión}

De los hallazgos de nuestro estudio destaca la mayor gravedad de la BA tanto en los menores de 3 meses como los VRS (+) en forma independiente por su mayor riesgo a ser hospitalizado. No encontramos correlación significativa entre factores ambientales y el número de casos, sólo entre aumento de PM2.5 y menor temperatura. El análisis de autocorrelación nos mostró que sólo la variable número de casos era estacional, es decir que tiene un patrón cíclico con variabilidad diaria. Como predictor de riesgo de hospitalización, sólo encontramos que lo aumentaba el tabaquismo intradomiciliario y la menor edad del niño. No se encontró relación significativa entre las variables en el tiempo.
Históricamente se ha responsabilizado a la contaminación ambiental al aumento de incidencia de patología respiratoria y mortalidad por esta causa, siendo conocido el caso de Londres en 1952 en que la morbimortalidad aumentó hasta 4 veces durante un período de altos niveles de contaminación ${ }^{12}$. Muchos son los estudios epidemiológicos que dan cuenta de esta asociación. En una revisión de los efectos en la salud de la contaminación aérea se puede destacar los efectos del $\mathrm{O}_{3}, \mathrm{SO}_{2}$ con disminución de la función pulmonar, hiperreactividad de la vía aérea, inflamación pulmonar, aumento de la permeabilidad vascular pulmonar, broncoconstricción, incremento de síntomas respiratorios, agregándose a éstos el incremento de la mortalidad con el aumento de PM10 y CO con disminución de la capacidad de ejercicio ${ }^{14,15}$. Estudios epidemiológicos, apoyados por estudios de metaanálisis de ellos, consistentemente demuestran una asociación entre contaminación por partículas y exacerbaciones en pacientes con enfermedades respiratorias y aumento de muertes por enfermedad respiratoria y cardiovascular. Se sugiere la hipótesis que las partículas ultra-finas provocan inducción de inflamación de la vía aérea, adhesión de moléculas endoteliales y leucocitos en la sangre, alteración de la coagulabilidad sanguínea, alteración de la actividad eléctrica cardíaca que podrían explicar también el incremento de las muertes por problemas cardiovasculares ${ }^{16,17}$. Sin embargo, hay estudios en que esta asociación entre el aumento de PM10 y el de los síntomas respiratorios la encuen- 
tran en forma débil o no la encuentran ${ }^{18,19}$. En nuestro estudio no encontramos aumento de la consulta u hospitalización por BA en asociación con aumento de PM10 o PM2.5, sí un mayor riesgo de hospitalización en los pacientes con infección por VRS, menores de 3 meses y que habitan en ambientes contaminados por el humo del tabaco.

Estudios previos en niños han demostrado asociación entre el aumento de la mortalidad con episodios muy severos de alta contaminación, baja de la frecuencia de hospitalización con disminución de los niveles de PM10 o aumento de hospitalización o consultas por laringotraqueobronquitis con aumento de los niveles de $\mathrm{NO}_{2}$, así también como tos y secreciones bronquiales con exposición crónica a contaminantes ambientales, disminución de función pulmonar y exacerbaciones de asma y contaminación aérea ${ }^{20-22}$, como también la susceptibilidad a la contaminación aérea que tienen los niños con hiperreactividad bronquial y altas concentraciones de Ig E sérica o síntomas respiratorios crónicos ${ }^{4,5}$. En una revisión de los estudios de serie de tiempo para mortalidad relacionada con PM10 se encontró que éstos confirman que una asociación de contaminación aguda con mortalidad puede ocurrir ${ }^{8}$, como también con el número de hospitalizaciones ${ }^{6}$. Buchdahl y cols, encontraron que en forma independiente a la estación del año y temperatura, las fluctuaciones de las concentraciones de $\mathrm{O}_{3}$ y $\mathrm{SO}_{2}$ están fuertemente asociadas a consulta en el SU por episodios de sibilancias ${ }^{23}$. En nuestro estudio no encontramos asociación de consulta u hospitalización por BA y estos factores ambientales.

Con respecto a la relación específica del efecto de la contaminación ambiental con el VRS o con BA hay escasa literatura. En un estudio del efecto del $\mathrm{O}_{3}$ sobre macrófagos alveolares con respecto a su susceptibilidad a infección por VRS y su producción de citoquinas, se observó que tenían una producción menor de IL-6 en comparación a los controles ${ }^{24}$. A nivel nacional, en el área norte de Santiago, se realizó un trabajo que estudió el impacto de la epidemia de virus respiratorios, el frío y la contaminación aérea con la demanda de atención pediátrica. En este estudio tampoco se encontró una relación de ésta con aumento de PM10 y los investigadores postularon que even- tualmente podría encontrarse con PM2.5, lo que nuestro estudio no pudo demostrar al evaluar este parámetro. En relación a la baja temperatura, se demostró que tenía un efecto discreto en la demanda asistencial, siendo lo que más impactó en la demanda la presencia de virus respiratorios en la comunidad ${ }^{2}$.

Nuestro trabajo tiene algunos defectos, siendo el principal de ellos el número de casos, ya que para poder evaluar el efecto ecológico de una variable hay que contar con 25 casos al día y si es posible, con la información de toda la Región Metropolitana. Además, con respecto a la contaminación intradomiciliaria, contamos con la información por encuesta telefónica de tabaco y elementos de calefacción, pero no obtuvimos una medición objetiva de ésta. A su vez, como aspectos positivos, creemos que el trabajo aborda un tema interesante, con escasa literatura previa, que es la de la relación del primer episodio de BA con contaminación ambiental, en que se cuenta con los datos individuales de los pacientes, con información de los pacientes tanto de consulta ambulatoria, urgencia y hospitalizados. Por otra parte, en el aspecto ambiental por primera vez se cuenta con la información de PM2.5, que años anteriores no se medía y con la presencia de datos pluviométricos. A futuro, sería importante poder contar con la información de más centros de atención en este mismo período de tiempo, recolectando datos de un mayor número de casos y así probablemente las tendencias que se observan de manera inicial, pero que no resultan ser significativas en nuestro estudio, puedan confirmar los resultados esperados.

En resumen, nuestro estudio destaca el mayor riesgo de hospitalizarse de lactantes con bronquiolitis aguda que son menores de 3 meses, con VRS (+) y que presentan tabaquismo pasivo intradomiciliario. Se encontró relación entre PM10, PM2.5 y temperaturas bajas como también entre PM2.5 y PM10. Creemos que es fundamental realizar un estudio multicéntrico colaborativo para contar con un número mayor de pacientes que permita obtener resultados más representativos a nivel nacional para determinar la real correlación entre contaminación ambiental y la presencia de bronquiolitis aguda. 


\section{REFERENCIAS}

1. DenNy FW, Clyde WA JR. Acute lower respiratory tract infections in non hospitalized children. J Pediatr 1986; 108: 635-46.

2. Avendaño L, Céspedes A, Stecher $X$, Palomino MA. Influencia de virus respiratorios, frío y contaminación aérea en la infección respiratoria aguda baja del lactante. Rev Méd Chile 1999; 127: 1073-8.

3. Kim P, Musher D, Gifzen P, Rodríguez-Barradas M, NAHM W, WRIGHT CE. Association of invasive pneumococcal disease with season, atmospheric condition, air pollution, and the isolation of respiratory viruses. Clini Infect Dis 1996; 22: 100-10.

4. Boezen HM, van der Zee SC, Postma DS, Vonk JM, GerRitsen J, Hoek G et al. Effects of ambient air pollution on upper and lower respiratory symptoms and peak expiratory flow in children. Lancet 1999; 353: 874-8.

5. van der Zee S, Hoek G, Boezen HM, Schouten JP, van WijNen JH, BRUNekreEF B. Acute effects of urban air pollution on respiratory health of children with and without chronic respiratory symptoms. Occup Environ Med 1999; 56: 802-12.

6. Atkinson RW, Ross Anderson H, Sunyer J, Ayres J, Baccini M, VonK JM ET aL. Acute effects of particulate air pollution on respiratory admissions. Results from APHEA 2 project. Am J Respir Crit Care Med 2001; 164: 1860-6.

7. Lewis SA, Corden JM, Forster GE, Newlands M. Combined effects of aerobiological pollutants, chemical pollutants and meteorological conditions on asthma admissions and A \& E attendances in Derbyshire UK, 1993-96. Clin Exp Allergy 2000; 30: 1724-32.

8. THURSTON GD. A critical review of PM10 -mortality time- series studies. J Expo Anal Environ Epidemiol 1996; 6: 3-21.

9. LeBowitTz MD. Epidemiological studies of the respiratory effects of air pollution. Eur Respir J 1996; 9: 1029-54.

10. BuRR M. Indoor Air Pollution and the Respiratory Health of Children. Pediatr Pulmonol 1999; S18: 3-5.
11. Katz MH. Multivariable Analysis. A practical guide for clinicians. Cambridge University. Press 2001.

12. SCHWARTZ J, MARCUS A. Mortallity and air pollution in London: a time series analysis. Am J Epidemiol 1990; 131: 185-94.

13. WeInMANN GG. An update on air pollution. Curr Opin Pulm Med 1996; 2: 121-8.

14. Committee of the Environmental and Occupational Health Assembiy of the American Thoracic Society. Health Effects of Outdoor Air Pollution. Am J Respir Crit Care Med 1996; 153: 3-50.

15. Be山o S, Oyarzún M. Efectos Adversos para la Salud de los Contaminantes Atmosféricos. Rev Chil Enf Respir 1991; 7: 198-205.

16. Seaton A, MacNee W, Donaldson K, Godden D. Particulate air pollution and acute health effects. Lancet 1995; 345: 176-8.

17. Utel MJ, Frampton MW. Acute health effects of ambient air pollution: the ultrafine particle hypothesis. J Aerosol Med 2000; 13: 355-9.

18. Roemer W, Hoek G, Brunekreef B, Clench-Aas J, Forsberg B, Pekranen J, Schutz A. PM10 elemental composition and acute respiratory health effects in European children (PEACE project). Eur Respir J 2000; 15: 553-9.

19. Osunsanya T, Prescott G, Seaton A. Acute respiratory effects of particles: mass or number? Occup Environ Med 2001; 58: 154-9.

20. SÉGAL C. Health Effects of Urban Outdoor Air Pollution in Children. Current Epidemiological Data. Pediatr Pulmonol 1999; 18S: 6-8.

21. BATES DV. The effects of air pollution on children. Environ Health Perspect 1995; 103 Suppl 6: 49-53.

22. Nicolai T. Air Pollution and Respiratoryr Disease in Children: What is the Clinically Relevant Impact? Pediatr Pulmonol 1999; 18: 9-13.

23. Buchdahl R, Parker A, Stebbings T, Babiker A. Association between air pollution and acute childhood wheezy episodes: prospective observational study. BMJ 1996; 312: 661-5.

24. Soukup J, Koren Hs, BeCKer S. Ozone effect on respiratory syncytial virus infectivity and cytokine production by human alveolar macrophages. Environ Res 1993; 60: 178-86. 